\title{
BCS-IRSG Workshop on Corpus Profiling
}

\author{
London \\ 18 October 2008
}

Editors:

Anne De Roeck
Dawei Song
Udo Kruschwitz 


\section{Abstract}

We aim to bring together people from different research communities interested in exploring how corpus characteristics affect the behaviour of techniques in information retrieval and natural language processing, and to set out a roadmap for a shared research agenda.

It is well known in NLP and IR that the effectiveness of a technique depends on both the data on which it is deployed and its match with the task at hand. In 1973, Spärck-Jones attributed differing degrees of success at automatic classification to differences in dataset characteristics. Since Croft and Harper (1979), IR performance has repeatedly been related to collection size and other features, though no upper bound has been found.

The importance of data and task dependencies has been highlighted in IR, anaphora resolution, automatic summarization and recently, in word sense disambiguation. Many web/enterprise web retrieval systems rely on URL properties, link graph properties, click streams, and so on, with performance dependent on the degree to which this evidence is present and meaningful in a particular corpus.

This conference was sponsored by

\section{$\underline{B C S ~ I R S G}$}




\section{Full Synopsis}

The Workshop on Corpus Profiling for Information Retrieval and Natural Language Processing took place in London, in October 2008, in conjunction with IliX2008. Our aim was to bring together people from different research communities interested in exploring how specific properties of a corpus or collection affect the behaviour of techniques in Information Retrieval (IR) and Natural Language Processing (NLP), and to start mapping out a shared research agenda. These eWiCs Proceedings capture the final versions of papers presented at the workshop. 


\section{Editors}

This conference was edited by:

Anne De Roeck - (Open University)

Dawei Song - (Robert Gordon University Aberdeen)

Udo Kruschwitz - (University of Essex) 


\section{Papers:}

\section{Session 1: Genre}

Malcolm Clark, Ian Ruthven and Patrik O'Brian Holt Genre analysis of structured emails for corpus

profiling $\quad$ http://dx.doi.org/10.14236/ewic/IRSG2008.1

V. F. Berninger, Yunhyong Kim and Seamus Ross Building a document genre corpus: a profile of the KRYS I corpus http://dx.doi.org/10.14236/ewic/IRSG2008.2

Foaad Khosmood and Robert A. Levinson Automatic Natural Language Style Classification and Transformation http://dx.doi.org/10.14236/ewic/IRSG2008.3

\section{Session 2: Words}

Mark Greenwood and Goran Nenadic Lexical Profiling of Existing Web Directories to Support Finegrained Topic-Focused Web Crawling http://dx.doi.org/10.14236/ewic/IRSG2008.4

Neil Cooke and Lee Gillam Distributional Lexical Semantics for Stop Lists http://dx.doi.org/10.14236/ewic/lRSG2008.5 